socioeconomic status youth, admitted in Khon Kaen Hospital due to severe injury from traffic accident and holistic care Multidisciplinary team provided. Sample were 25 traffic accident 23 men and 2 women average ages was 41 (min-max= 6-87 years, Mode $=25$ ) which were 12 spinal cord 4 head and other organs injury patients. Quality of life was measure in 2004 by brief Thai WHO quality of life and found medium to low quality of life score $76 \%$ then PDCA schemes to empower using four attributes of empowerment concept.

Results were as follows: A traffic accident disable club established. Member consisted of more than 48 members. They could be able to live, more, independently. Three of wheelchair person married (on wheelchair)and having good family. Two bed ridden SCI could be able to get out of bed. Six person engaged new career. Twelves (48.0\%) are core of the team and consistently join the clubs routine activities. Others are also actively return their experience to society occasionally but keep in touch. Their routine activities are (1).Joining multidisciplinary health team visiting new traffic accident injured patients in hospital and at the patients home. (2). traffic accident prevention campaigning in Khon Kaen Hospital every morning, twice a month in schools and occasionally in publics. (3).Lecturer in any meeting concerning traffic accident prevention and campaign in public meeting colleges and universities.

\title{
0065 EFFECT OF EMPOWERMENT ON QUALITY OF LIFE AND SOCIAL ABILITY OF ROAD TRAFFIC ACCIDENT DISABLES IN KHON KAEN HOSPITAL
}

PShawong*, JChantarasiri, SKulleub,TKaesri,TSrilamai,TManeerat Correspondence: Khon Kaen Hospital, 54-56 Srichan Rd. Khon Kaen, 40000, Thailand

10.1136/ip.2010.029215.65

This Action Research aimed to develop quality of life of the traffic accident disables who mostly were less opportunity 